\title{
Trastuzumab treatment after progression in HER2-positive metastatic breast cancer following relapse of trastuzumab-based regimens: a meta-analysis
}

This article was published in the following Dove Press journal:

Cancer Management and Research

\author{
Yiqun Han' \\ Jiayu Wang' \\ Weiming Liu $^{2}$ \\ Peng Yuan' \\ Qing $\mathrm{Li}^{1}$ \\ Pin Zhang' \\ Fei Ma' \\ Yang Luo' \\ Ying Fan' \\ Shanshan Chen' \\ Ruigang Cai' \\ Qiao Li' \\ Binghe $\mathrm{Xu}{ }^{\prime}$ \\ 'National Cancer Center/National \\ Clinical Research Center for Cancer/ \\ Cancer Hospital, Chinese Academy of \\ Medical Sciences and Peking Union \\ Medical College, Beijing, People's \\ Republic of China; ${ }^{2}$ Department of \\ Critical Care Medicine, China \\ Rehabilitation Research Center, Beijing, \\ People's Republic of China
}

Background: This meta-analysis assessed the safety and effectiveness of retreatment with trastuzumab in patients with human epidermal growth factor receptor 2 (HER2)-positive metastatic breast cancer (HER2+MBC).

Materials and methods: Randomized controlled trials (RCTs) and cohort studies that compared the clinical outcomes of continuation and termination of trastuzumab treatment in HER2+MBC after failure of trastuzumab-based regimens were analyzed. Pooled estimates of time to progression (TTP) survival, overall survival (OS), the incidence of adverse events and central nervous system (CNS) perturbations were determined.

Results: Four RCTs and six cohort studies with 2,409 patients were identified. The continuation of trastuzumab presented a statistical significance in prolonging TTP (HR 0.88; 95\% CI: $0.82-0.94 ; P<0.000$ ) and OS (HR 0.87; 95\% CI: 0.82-0.93; $P<0.000$ ). Furthermore, retreatment with trastuzumab did not add to the risk of cardiac events (relative risk, 2.48; 95\% CI: $0.86-7.15)$ or the incidence of CNS metastasis $(P=0.83)$.

Conclusion: Our findings confirm the clinical benefits and safety of retreatment therapy with trastuzumab for HER2-positive patients with metastatic cancer of the breast that had progressed during trastuzumab-based treatment regimens.

Keywords: trastuzumab, retreatment, HER2-positive cancer, metastatic cancer of the breast, progression therapy

\section{Background}

Cancer of the breast is a global cause of death in women. ${ }^{1}$ About $20-30 \%$ of patients with breast cancer characteristically overexpress human epidermal growth factor receptor 2 (HER2), which leads to aggressive progression of the disease. The monoclonal antibody trastuzumab selectively interacts with an extracellular site on HER2. Since the definite antitumor activity of trastuzumab was identified by Baselga in $1996,{ }^{2}$ it has been widely used in clinical practice and suggested a favorable compatibility in breast cancer treatment at every stage.

Treatment for HER2+MBC beyond progression is a key challenge. ${ }^{3}$ CLEOPATRA reported that pertuzumab in combination with trastuzumab and docetaxel significantly prolonged progression-free survival (PFS) and overall survival (OS) median times. ${ }^{4}$ Trastuzumab emtansine (T-DM1), an antibody-drug conjugate in which trastuzumab is covalently bound to the antimicrotubule polymerization blocker DM1, ${ }^{5}$ has been proven
National Cancer Center/National Clinical Research Center for Cancer/Cancer Hospital, Chinese Academy of Medical Sciences and Peking Union Medical College, No. 17 Panjiayuan Nanli,

Chaoyang District, Beijing 10002I, People's Republic of China

Tel +86 I58 I008 0308

Fax +86 1087788120

Email wangjiayu7887@outlook.com 
to improve OS compared to a combination of capecitabine + lapatinib in HER2-positive patients whose advanced breast cancer is advanced. ${ }^{6}$ Based on clinical results, the National Comprehensive Cancer Network committee has recommended a combination of trastuzumab + pertuzumab + taxanes as standard initial therapy, and T-DM1 monotherapy as second-line therapy for HER2-positive metastatic breast cancer (HER2+MBC) after trastuzumab-based regimens have been shown to be ineffective. However, the clinical benefit of these novel drugs in HER2+MBC remains controversial. ${ }^{1}$ Furthermore, these drugs are not available worldwide, which largely limit the choices for the treatment of this serious condition.

Unlike the established paradigm of stopping and switching drugs or interventions after disease progression, the practice of continuation of trastuzumab has always been considered to be acceptable during the last decade. Indeed, preclinical findings have shown that consistent anti-HER2 treatment can effectively inhibit the proliferation of cancer cells. ${ }^{7}$ Retrospective studies further suggested that the prolonged treatment with trastuzumab would definitely provide significant clinical benefit. ${ }^{8-10}$ However, the use of trastuzumab-containing regimens beyond disease progression has been questioned, especially due to the resistance to trasutuzmab and adverse events (AEs), especially cardiac toxicities. ${ }^{11}$ Thus, we carried out a meta-analysis to evaluate the safety and efficacy of retreatment with trastuzumab for patients beyond progression in HER2+MBC after the failure of previous trastuzumab therapy.

\section{Materials and methods}

\section{Literature search strategy}

Randomized controlled trials (RCTs) and cohort studies up to February 2018 were gathered through searches of Pubmed, Embase, Cochrane Library, Clinical Trials, Sinomed, CNKI, and VIP information. We used the keywords of trastuzumab, HER2-positive, metastatic breast cancer, and MeSH terms including breast neoplasm, breast carcinoma, ErbB-2 to screening investigations in Pubmed. We also utilized the keywords of trastuzumab, HER2positive, metastatic breast cancer, chemotherapy to search in Cochrane Library and Embase. We also scrutinized the database and included Sinomed, CNKI, and VIP information. As for a supplementary counterpart, we analyzed reviews, conference reports, and other article types, which referred to retreatment with trastuzumab in HER2
$+\mathrm{MBC}$ patients and evaluated the trials cited in the present references according to implicit keywords. Moreover, the bibliographies of eligible articles were also manually screened to identify potential additional studies.

\section{Eligibility criteria}

The inclusive standards were: 1) patients $\geq 18$ years old with histologically or cytologically confirmed HER2 $+\mathrm{MBC}$ detected by immunohistochemistry or fluorescence in situ hybridization $2+/ 3+$ cancer type, 2) objective disease progression on prior trastuzumab-containing regimes, defined by RECIST or disease of the bone only, 3) RCTs or cohort studies, 4) experimental groups designed with trastuzumab-containing regimens or monotherapy with control groups treated identically other than with trastuzumab, 5) reports of specific OS, time to progression (TTP) survival, and/or AEs of cardiac safety.

Trials were excluded if patients were recruited without confirmed metastatic sites or were not pretreated with trastuzumab; the follow-up period was unclear, the withdrawal rate was $>10 \%$, and authors did not report TTP, OS, and/or AEs of cardiac safety. Considering the limited number of subjects and the rare incidence of AEs, we did not regard the methodology of study design as one of the exclusive standards. Both RCTs and observational studies that met the inclusive standards were all adopted and stratified into subgroups.

\section{Data extraction}

We recorded the following items: first author and year, study design, recruitment time, sample size, and administrative details of each group. The primary endpoint was TTP and OS. The definition of TTP was the interval between the initiation of second-line therapy that included trastuzumab and the degree of disease progression. Since the meanings were similar, we did not further distinguish PFS and TTP. The definition of OS was the time from the initiation of second-line therapy including trastuzumab to the death of a patient. Secondary endpoints were the incidence of AEs and central nervous system (CNS) metastasis. Quality of life (QOL) scores were also analyzed.

To assess the efficacy of retreatment with trastuzumab, survival analysis was implemented. We extracted the HR of TTP and OS reported in each trial. Based on the statistical methods introduced in the treatise of Tinerney and Williamson, log HR and SE were calculated to determine the synthesized HR and its $95 \%$ CI. ${ }^{12}$ To assess the safety of this practice, we extracted the incidence of AEs, among 
which were mainly cardiac events. The dichotomous results were used to calculate the values for the risk ratio (RR) and 95\% CI. Given the fact that the data of QOL were rare, we decided to make a descriptive analysis rather than pooling it.

\section{Quality assessment}

Two investigators accomplished this section independently. In view of the different designs of included studies, we used different methods for evaluating their quality. On the one hand, the Cochrane Collaboration's tool was adopted to assess the risk of bias in RCTs. On the other hand, the Newcastle-Ottawa Scale was utilized for cohort studies. Quality assessment results are shown in Figures 1 and 2.

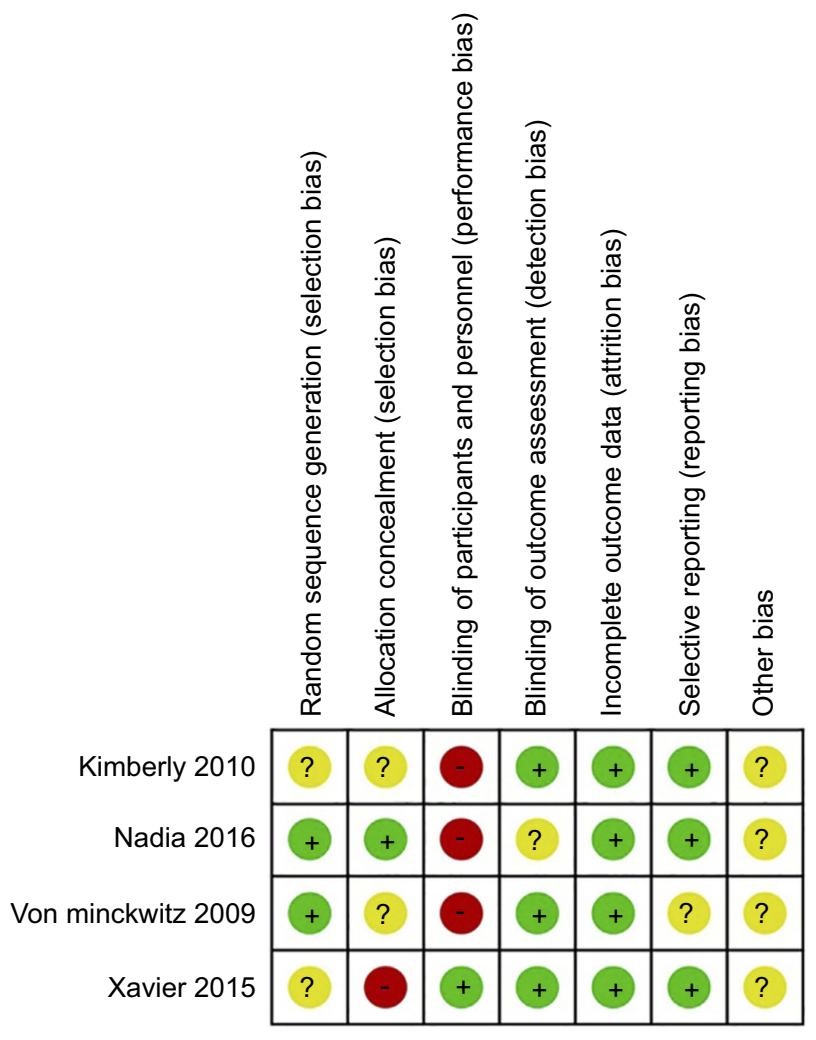

Figure I Review authors' judgments about each risk of bias item for RCTs. Abbreviation: RCTs, randomized controlled trials.

\section{Statistical analysis}

Data were extracted from articles by two independent investigators. The HR as the survival type outcome was used to measure TTP and OS. All-inclusive papers clearly demonstrated their corresponding data. We directly extracted the HRs and their 95\% CI as reported. RR was utilized to measure the dichotomous outcomes including the incidence of AEs and CNS metastasis.

We assessed heterogeneity using the $\mathrm{Chi}^{2}$ test and the $I^{2}$ statistic. ${ }^{13}$ We classified $I^{2}$ values $<50 \%$ as having homogeneity and a fixed-effect model was well accepted. An $I^{2}$ value $>50 \%$ predicted potential heterogeneity. If heterogeneity existed, subgroup analysis was used to weaken its effects. Else, we synthesized the results with a randomized effect model if no definite heterogeneity was detected.

\section{Results}

\section{Characteristics of included clinical trials}

Figure 3 shows a schematic of the study selection. Initially, 1,236 references were identified. Finally, $4 \mathrm{RCTs}^{14-17}$ and 6 cohort studies ${ }^{18-23}$ were included that comprised of 2,409 patients who met the standard. A total of 1,293 patients were randomly allocated to the trastuzumab group and 1,116 patients were assigned to the control group. All participants were female with HER2 $+\mathrm{MBC}$, which progressed despite treatment with trastuzumab regardless of age, race, menopausal status, ER, PR status, or prior metastatic sites. The patients' ages varied from 51 to 59 years. Basic information about the included studies is shown in Table 1.

\section{Efficacy}

TTP was obtained from 4 RCTs and 1 cohort study. The HRs about TTP in RCTs were extracted and pooled. Among these trials, trasutuzmab-containing regimens (trasutuzmab + capecitabine $v s$ lapatinib + capecitabine/capecitabine monotherapy, trasutuzmab vs lapatinib vs lapatinib monotherapy) demonstrated a statistical improvement in TTP. Nadia showed that afatinib + vinerolbine did not improve TTP in HER2+MBC

\begin{tabular}{|c|c|c|c|c|c|c|c|c|c|}
\hline \multirow[t]{2}{*}{ Studies } & \multicolumn{4}{|c|}{ Selection } & \multirow[t]{2}{*}{ Comparability } & \multicolumn{3}{|c|}{ Outcome assessment } & \multirow[t]{2}{*}{ Score } \\
\hline & 1 & 2 & 3 & 4 & & 1 & 2 & 3 & \\
\hline Cancello $2009^{[17]}$ & * & * & * & * & & * & * & * & $\star * \star * * * *$ \\
\hline Exra $2010^{[18]}$ & * & * & * & * & & * & * & * & $* * * * * * *$ \\
\hline Hammerman $2015^{[19]}$ & * & * & * & * & ** & & * & * & $\star * * * * * * *$ \\
\hline Jackisch 2014 [20] & * & * & & * & ** & * & * & * & $* * * * * * *$ \\
\hline Montemurro $2008^{[21]}$ & * & * & * & * & * & * & & * & $* * * * * * *$ \\
\hline Negri $20144^{[22]}$ & * & * & * & * & & * & * & * & $* * * * * * *$ \\
\hline
\end{tabular}

Figure 2 Quality indicators from the Newcastle-Ottawa sale for cohort studies. Notes: In this evaluation form, *represents one point, ** represents two points, *******or more indicates that the quality of the article is high and credible out of 9 points. 


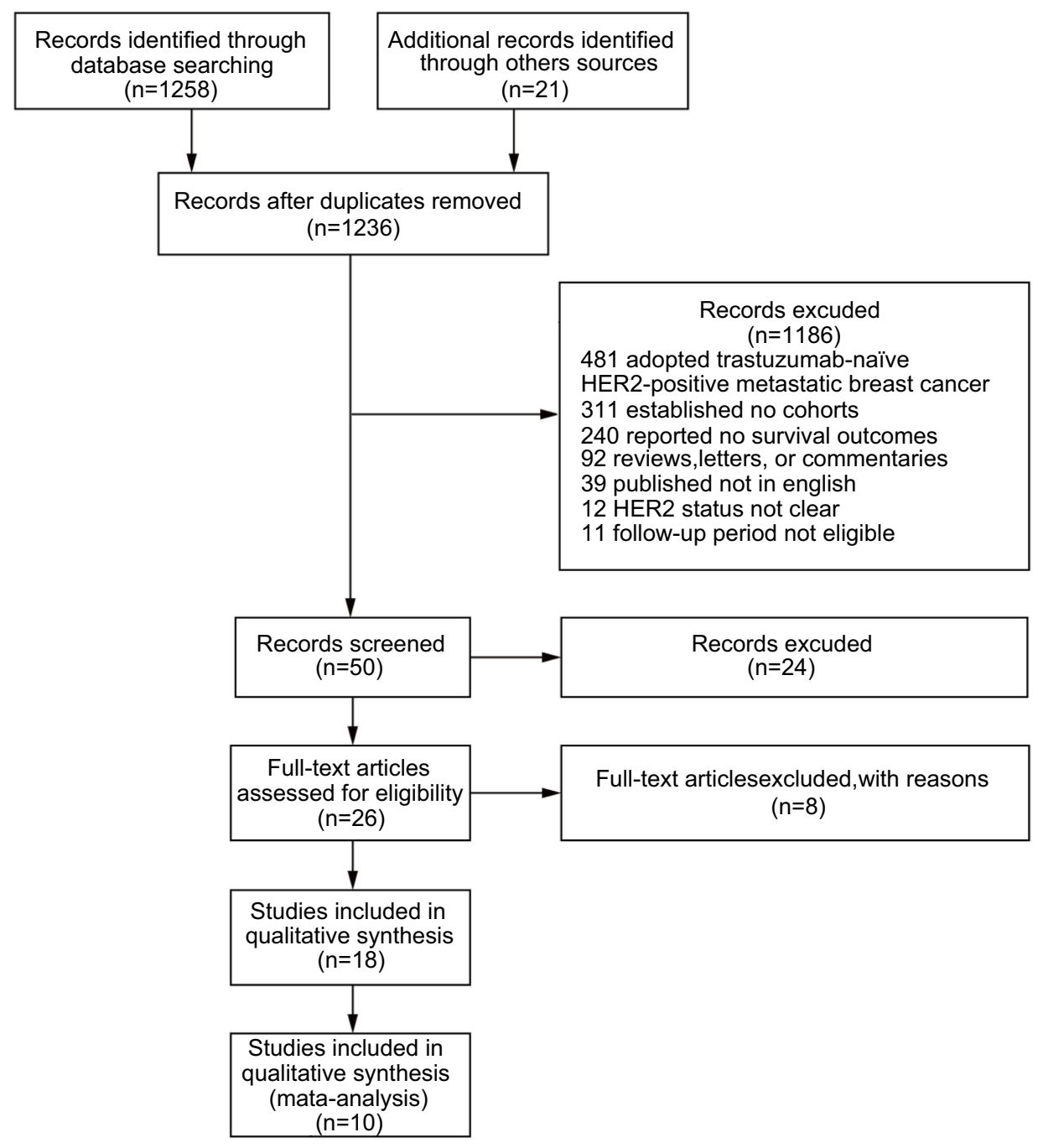

Figure 3 The process of the study selection.

Table I Characteristics of inclusive trials

\begin{tabular}{|c|c|c|c|c|c|}
\hline \multirow[b]{2}{*}{ Author year } & \multirow[b]{2}{*}{ Study design } & \multirow[b]{2}{*}{ Recruitment time } & \multicolumn{2}{|c|}{ Patients (n) } & \multirow[b]{2}{*}{ Exposure to trastuzumab } \\
\hline & & & T-group & C-group & \\
\hline Cancello $2009^{17}$ & Cohort & $2000.07-2007.01$ & 59 & 23 & $\mathrm{~T}+\mathrm{CT}$ \\
\hline Extra $2010^{18}$ & Cohort & $2002.01-2005.03$ & 107 & 70 & $\mathrm{~T}+\mathrm{CT}(94 \%) / \mathrm{ET}(22 \%)$ \\
\hline Hammerman $2015^{19}$ & Cohort & $2010.01-2013.08$ & 64 & 93 & $\mathrm{~T}+\mathrm{CT}(80 \%) \mathrm{ET}(20 \%)$ vs L+Cap \\
\hline Jackisch $2014^{20}$ & Cohort & $2000.01-2010.06$ & 261 & 157 & $\mathrm{~T}+\mathrm{CT}(80 \%) / \mathrm{ET}$ or MT $(18 \%)$ \\
\hline Kimberly $2010^{13}$ & $\mathrm{RCT}$ & $2005.1 I-2006.1 I$ & 148 & 148 & $T+L$ vs $L$ \\
\hline Montemurro $2008^{21}$ & Cohort & $2002.12-2007.03$ & 83 & 112 & $\mathrm{~T}+\mathrm{CT}$ \\
\hline Nadia $2016^{14}$ & $\mathrm{RCT}$ & $2010.08-2013.04$ & 339 & 169 & $A+V$ vs $T+V$ \\
\hline Negri $2014^{22}$ & Cohort & $2006.08-2009.12$ & 96 & 197 & NA \\
\hline von Minckwitz $2009^{16}$ & $\mathrm{RCT}$ & 2003.09-2007.06 & 78 & 78 & T+Cap vs Cap \\
\hline Xaiver $2015^{15}$ & Cohort & $2009.04-2012.06$ & 58 & 69 & $T+$ Cap vs $L+C a p$ \\
\hline
\end{tabular}

Abbreviations: T-group, trastuzumab group; C-group, control group; CT, chemotherapy; ET, endocrine therapy; A, afatinib; V, vilrobine; NA, not available; Cap, capecitabine; L, lapatinib; MT, monotherapy trastuzumab; RCTs, randomized controlled trials.

patients $(\mathrm{HR}=1.10 ; 95 \%$ CI: $0.86-1.41, P<0.000) .{ }^{15}$ After picking up the reciprocal and synthesizing the results, we found the pooled HR for TTP was 0.88 (95\% CI: 0.82-0.94) without heterogeneity $\left(I^{2}=0\right)$. The results are shown in Figure 4. Montemurro suggested that continuation of trastuzumab did not seem to reduce the time to second disease 


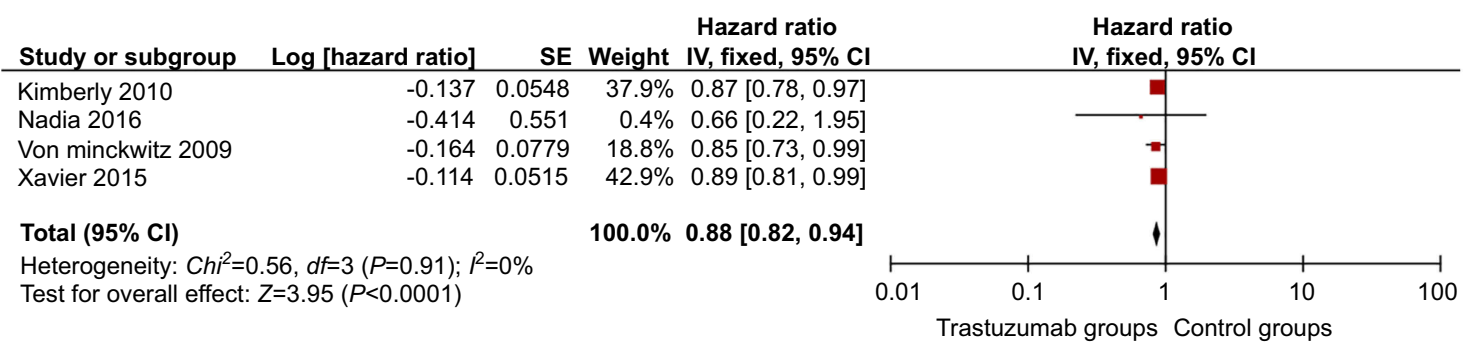

Figure 4 Forest plot: time to progression.

progression ( $\mathrm{HR}=0.753$; 95\% CI: $0.548-1.035, P<0.000) .{ }^{22}$ To avoid the heterogeneity of methodological limitation, we did not add these results to the pooled data.

Three RCTs and 5 observational studies reported OS values for retreatment with trastuzumab and we directly extracted them. To further rule out substantial heterogeneity, we stratified the data according to the study design. Both the two subgroups showed a statistically significant difference. The pooled HR was 0.85 (95\% CI: 0.77-0.94) for RCTs and 0.89 (95\% CI: 0.82-0.96) for observational studies, respectively. Although the heterogeneity for the latter studies was severe, we regarded it as the consequence of the methodology and lack of randomization. The test for differences between the study design subgroups was not statistically significant $(P=0.46)$. The results are shown in Figure 5 .

QOL has been taken into account as an important factor to evaluate the effectiveness of cancer chemotherapy. It is an equally important factor for treatment decisions. In these adopted trials, just one clinical trial conducted a QOL assessment. Based on FACT-B questionnaire (version 4),
Kimberly considered that there were no statistical differences in possible changes from the baseline in the Functional Assessment of Cancer Therapy (FACT) - general or FACT-B total scores or in the trial outcome index. ${ }^{14}$ However, no detailed data were shown in the articles.

\section{Safety}

The most concerning AEs during trastuzumab therapy were cardiac events. Von Minckwitz reported severe cardiac events (grade 3-4) that were observed in four patients in the capecitabine + trastuzumab group and a left ventricular ejection fractions(LVEF) $<40 \%$ was reported in one other patient. ${ }^{17}$ In contrast, two patients were observed with severe cardiac events and none with an LVEF decrease during capecitabine monotherapy. In LUX-Breast 1, only common AEs (>1\%) were listed and one patient in the trastuzumab-containing regimen was observed with a grade $\geq 3$ cardiac event (grade 3 hypertension). ${ }^{15}$ Kimberly reported eight patients undergoing trasutuzmab-containing therapy with a severe LVEF decrease ( $\geq 20 \%$ ) and three patients similar to the control group. ${ }^{14}$ Due to

\begin{tabular}{|c|c|c|c|c|c|c|c|}
\hline Study or subgroup & Log [hazard ratio] & SE & Weight & $\begin{array}{l}\text { Hazard ratio } \\
\text { IV, fixed, } 95 \% \mathrm{CI}\end{array}$ & $\begin{array}{r}\text { Hazard } \\
\text { IV, fixed, }\end{array}$ & $\begin{array}{l}d \text { ratio } \\
, 95 \% \mathrm{CI}\end{array}$ & \\
\hline \multicolumn{8}{|c|}{ 1.10.1 randomized controlled trials } \\
\hline Kimberly 2010 & -0.125 & 0.0769 & $15.6 \%$ & $0.88[0.76,1.03]$ & - & & \\
\hline Nadia 2016 & -0.246 & 0.0856 & $12.6 \%$ & $0.78[0.66,0.92]$ & 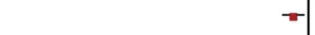 & & \\
\hline Von minckwitz 2009 & -0.117 & 0.104 & $8.5 \%$ & $0.89[0.73,1.09]$ & $=$ & & \\
\hline Subtotal $(95 \% \mathrm{Cl})$ & & & $36.7 \%$ & $0.85[0.77,0.94]$ & 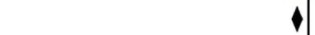 & & \\
\hline \multicolumn{8}{|c|}{$\begin{array}{l}\text { Heterogeneity: } C^{2} i^{2}=1.38, d f=2(P=0.50) ; P^{2}=0 \% \\
\text { Test for overall effect: } Z=3.28(P=0.001)\end{array}$} \\
\hline \multicolumn{8}{|c|}{ 1.10.2 observational studies } \\
\hline Cancello 2009 & -0.071 & 0.0924 & $10.8 \%$ & $0.93[0.78,1.12]$ & + & & \\
\hline Extra 2010 & -1.208 & 0.3176 & $0.9 \%$ & $0.30[0.16,0.56]$ & & & \\
\hline Hammerman 2015 & 0.201 & 0.1007 & $9.1 \%$ & $1.22[1.00,1.49]$ & & F & \\
\hline Jackish 2014 & -0.194 & 0.0522 & $33.8 \%$ & $0.82[0.74,0.91]$ & - & & \\
\hline $\begin{array}{l}\text { Negri } 2014 \\
\text { Subtotal (95\% Cl) }\end{array}$ & -0.102 & 0.1034 & $\begin{array}{r}8.6 \% \\
63.3 \%\end{array}$ & $\begin{array}{l}0.90[0.74,1.11] \\
\mathbf{0 . 8 9}[\mathbf{0 . 8 2}, \mathbf{0 . 9 6}]\end{array}$ & t & & \\
\hline \multicolumn{8}{|c|}{$\begin{array}{l}\text { Heterogeneity: } C h i^{2}=24.22, d f=4(P<0.0001) ; I^{2}=83 \% \\
\text { Test for overall effect: } Z=3.10(P=0.002)\end{array}$} \\
\hline \multirow{2}{*}{\multicolumn{4}{|c|}{$\begin{array}{l}\text { Total }(\mathbf{9 5 \%} \mathrm{Cl}) \\
\text { Heterogeneity: } C h i^{2}=26.13, d f=7(P=0.0005) ; P^{2}=73 \% \\
\text { Test for overall effect: } Z=4.46(P<0.00001) \\
\text { Test for subgroup differences: } C h i^{2}=0.54, d f=1(P=0.46\end{array}$}} & $0.87[0.82,0.93]$ & 1 & & \\
\hline & & & & ); $P^{2}=0 \%$ & \begin{tabular}{lcc} 
& \multicolumn{1}{l}{${ }_{0.1}^{+}$} & 1 \\
& Trastuzumab groups
\end{tabular} & $1 \frac{1}{1}$ & 100 \\
\hline
\end{tabular}

Figure 5 Forest plot: overall survival. 
the rare incidence of severe cardiac dysfunction, Xavier did not report these outcomes. ${ }^{16}$ The pooled estimate of RR was 2.48 (95\% CI: $0.86-7.15)$ and the $I^{2}$ value was 0 . It seems that retreatment with trastuzumab, from our statistical results, does not add to the risk of patients suffering from adverse cardiac events.

The most commonly reported AEs in inclusive trials were rash and diarrhea. Hence, we synthesized the reported incidence. Built on a random model, the pooled RR of diarrhea was 0.66 (95\% CI: 0.18-2.43) (shown in Figure 6) and that of rash was 0.33 (95\% CI: 0.06-1.93). No statistically significant differences were found in our results, and we believe that continuation of trastuzumab does not add to the risk of developing diarrhea or a rash.

Despite the fact that multiple HER2-targeted therapies have significantly improved in recent years, the prognosis of HER2+MBC and CNS metastasis remains a major treatment challenge. During the course of the disease, CNS metastasis occurs in up to $60 \%$ of HER $2+\mathrm{MBC}$ patients. Thus, we pooled the incidence of CNS metastasis in our synthesized analysis with the estimated RR of 1.08 (95\% CI: 0.53-2.22) and substantial heterogeneity (shown in Figure 7). The heterogeneity might be the outcome of the enrollment of inactive, asymptomatic brain metastases in LUX-breast $1 .{ }^{15} \mathrm{We}$ reasonably believe that the risk of CNS metastasis would not be increased due to retreatment with trastuzumab.

\section{Discussion}

This meta-analysis that included both RCTs and cohort studies confirmed the efficacy of continuation with trastuzumab therapy. Our results showed that continuous trastuzumab-containing regimens could improve TTP and OS after failure of (neo) adjuvant or cross-line trastuzumab treatments. Patients who received trastuzumab retreatment presented with good clinical tolerance. The results revealed that an extended period of trastuzumab treatment would not add to the risk of severe cardiac events or the incidence of CNS metastasis.

Trastuzumab primary or acquired resistance has become a key challenge in contemporary clinical practice. Continuous treatment durations with other ErbB-targeted agents are believed to provide better efficacy. ${ }^{12}$ However, Binghe Xu found that afatinib, a broader ErbB inhibitor, did not improve PFS or the objective response rate (ORR) and was also associated with shorter OS compared with trastuzumab. It should be made clear that some patients still remain sensitive to trastuzumab despite failure of prior trastuzumab therapy. ${ }^{14}$ Indeed, Luque-Cabal has proven that resistance to trastuzumab was not complete and could be reversed by acting as an escape route. ${ }^{23}$ A corpus of known resistance mechanisms, primarily obtained during preclinical studies, was partly divergent from clinical studies. Our results would provide more evidence for the need for further preclinical and clinical studies.

Previous research has been carried out to determine the efficacy of this practice. A systematic review that included 12 observational studies suggested that the weighted mean TTP was 23.66 weeks. ${ }^{24}$ Similar findings have reported that the weighted mean TTP was 7 months. ${ }^{25}$ The authors primarily explored and confirmed the clinical efficacy of continuation of trastuzumab beyond disease progression. However, there were no direct-pooled consequences about

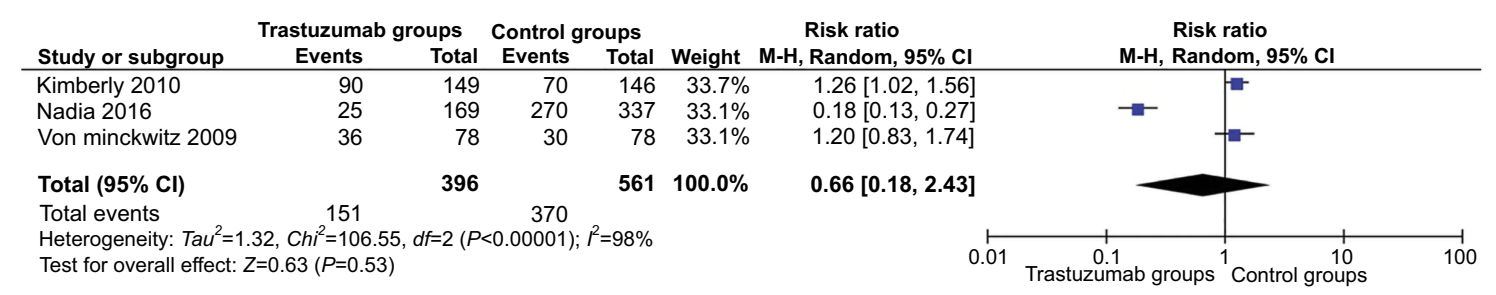

Figure 6 Forest plot: diarrhea.

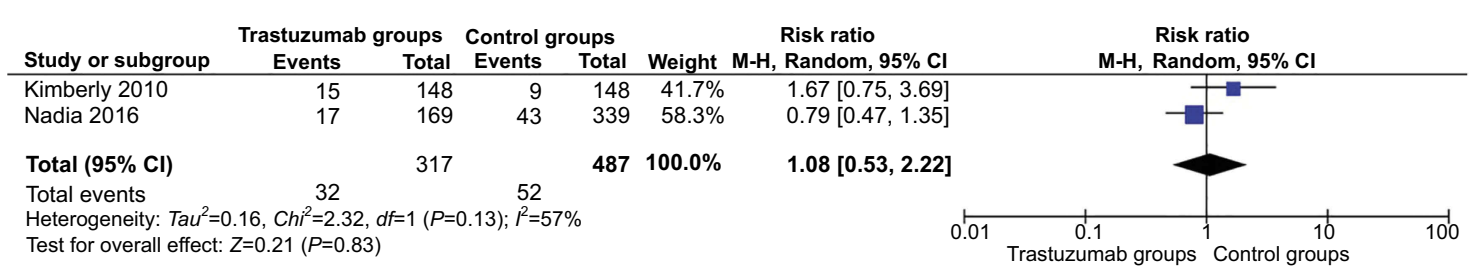

Figure 7 Forest plot: CNS metastasis.

Abbreviation: CNS, central nervous system. 
the incidence of AEs, especially of cardiac events, which were the main clinical concern about extended therapy with trastuzumab. So we cannot view the safety of retreatment with trastuzumab directly. Due to the limited number of RCTs and the nature of the observational study design, no synthesized results of time-to-event outcomes can be reliably reported.

Admittedly, there were some limitations in our findings. First, the molecular phenotype of breast cancer was not distinguished. Second, publication bias was not evaluated with Egger's test in view of the limited numbers and different types of inclusive studies. Considering that publication bias exists objectively and that the whole heterogeneity among them was favorable, we believe that publication bias would not have affected the reliability of our results. Third, some clinical trials are carried out without analysis, and we cannot pool the underlying results among them.

Numerous novel targeted agents have emerged in recent clinical trials and have been proven to provide substantial benefits. Pyrotinib (SHR-1258), an irreversible selective EGFR/HER2 dual inhibitor, has been shown to be effective in patients who had previously received treatment for HER2-positive metastatic disease. ${ }^{26}$ A randomized Phase II trial that compared capecitabine with lapatinib or pyrotinib showed that the ORR (78.5\% vs 57.1\%) and PFS (18.1 months vs 7 months) of the pyrotinib group were significantly better than in the counterpart. ${ }^{27}$ Trastuzumab deruxtecan (DS-8201) is an antibody-drug conjugate composed of an antibody against HER2, a link that can be cleaved by enzymes, and an agent that inhibits topoisomerase I. In a Phase I clinical trial of DS-8201, the OR was $40 \%$ and the rate of disease control $90 \%{ }^{28}$ With more novel targeted agents burgeoning, we look forward to promising advances in the therapy of HER2+MBC.

\section{Conclusions}

Our results revealed that continuation of trastuzumab therapy beyond progression could improve PFS and OS in HER2+MBC patients. In addition, this practice did not add to the risk of severe cardiac events. No statistical significance was found concerning the incidence of rash and diarrhea, and trastuzumab-containing regimes did not increase the risk for CNS metastasis after initiation of trastuzumab. According to the National Comprehensive Cancer Network panel, trastuzumab + pertuzumab and a cytotoxic agent (such as a taxane) may be considered the first-line therapy for HER2+MBC. T-DM1 also has substantial clinical benefits in HER2+MBC patients and is recommended as the standard second-line therapy for HER2+MBC. In some regions where these drugs are not available, retreatment with trastuzumab in HER2+MBC patients beyond disease progression remains an acceptable choice for oncologists; our findings provide convincing evidence for this assertion. Further research involving larger-scale, multicenter, randomized controlled clinical studies will be required to confirm our conclusions.

\section{Disclosure}

The authors report no conflicts of interest in this work.

\section{References}

1. Bray F, Ferlay J, Soerjomataram I, Siegel RL, Torre LA, Jemal A. Global cancer statistics 2018: GLOBOCAN estimates of incidence and mortality worldwide for 36 cancers in 185 countries. CA Cancer J Clin. 2018;68(6):394-424.

2. Baselga J, Tripathy D, Mendelsohn J, et al. Phase II study of weekly intravenous recombinant humanized anti-p185HER2 monoclonal antibody in patients with HER2/neu-overexpressing metastatic breast cancer. $J$ Clin Oncol. 1996;14(3):737-744. doi:10.1200/ JCO.1996.14.3.737

3. Balduzzi S, Mantarro S, Guarneri V, et al. Trastuzumab-containing regimens for metastatic breast cancer. Cochrane Database Syst Rev. 2014; Jun 12(6):Cd006242.

4. Swain SM, Baselga J, Kim SB, et al. Pertuzumab, trastuzumab, and docetaxel in HER2-positive metastatic breast cancer. $N$ Engl J Med. 2015;372(8):724-734. doi:10.1056/NEJMoa1413513

5. Niculescu-Duvaz I. Trastuzumab emtansine, an antibody-drug conjugate for the treatment of HER2+ metastatic breast cancer. Curr Opin Mol Ther. 2010;12(3):350-360.

6. Dieras V, Miles D, Verma S, et al. Trastuzumab emtansine versus capecitabine plus lapatinib in patients with previously treated HER2-positive advanced breast cancer (EMILIA): a descriptive analysis of final overall survival results from a randomised, open-label, phase 3 trial. Lancet Oncol. 2017;18(6):732-742. doi:10.1016/S14702045(17)30312-1

7. Slamon DJ, Godolphin W, Jones LA, et al. Studies of the HER-2/neu proto-oncogene in human breast and ovarian cancer. Science (New York, NY). 1989;244(4905):707-712. doi:10.1126/science.2470152

8. Stemmler HJ, Kahlert S, Siekiera W, Untch M, Heinrich B, Heinemann V. Prolonged survival of patients receiving trastuzumab beyond disease progression for HER2 overexpressing metastatic breast cancer (MBC). Onkologie. 2005;28(11):582-586. doi:10.1159/ 000088296

9. Waddell T, Kotsori A, Constantinidou A, et al. Trastuzumab beyond progression in HER2-positive advanced breast cancer: the Royal Marsden experience. Br J Cancer. 2011;104(11):1675-1679. doi:10.1038/bjc.2011.138

10. Metzger-Filho O, de Azambuja E, Procter M, et al. Trastuzumab re-treatment following adjuvant trastuzumab and the importance of distant disease-free interval: the HERA trial experience. Breast Cancer Res Treat. 2016;155(1):127-132. doi:10.1007/s10549-0153656-0

11. Nahta R, Yu D, Hung MC, Hortobagyi GN, Esteva FJ. Mechanisms of disease: understanding resistance to HER2-targeted therapy in human breast cancer. Nat Clin Pract Oncol. 2006;3(5):269-280. doi:10.1038/ncponc0509

12. Williamson PR, Smith CT, Hutton JL, Marson AG. Aggregate data meta-analysis with time-to-event outcomes. Stat Med. 2002;21 (22):3337-3351. doi:10.1002/sim.1303 
13. Higgins JP, Thompson SG, Deeks JJ, Altman DG. Measuring inconsistency in meta-analyses. $\mathrm{Br}$ Med J (Clin Res Ed). 2003;327 (7414):557-560. doi:10.1136/bmj.327.7414.557

14. Gelmon KA, Boyle FM, Kaufman B, et al. Lapatinib or trastuzumab plus taxane therapy for human epidermal growth factor receptor 2-positive advanced breast cancer: final results of NCIC CTG MA.31. J Clin Oncol. 2015;33(14):1574-1583. doi:10.1200/JCO.2014.56.9590

15. Harbeck N, Huang CS, Hurvitz S, et al. Afatinib plus vinorelbine versus trastuzumab plus vinorelbine in patients with HER2-overexpressing metastatic breast cancer who had progressed on one previous trastuzumab treatment (LUX-Breast 1): an open-label, randomised, phase 3 trial. Lancet Oncol. 2016;17 (3):357-366. doi:10.1016/S1470-2045(15)00540-9

16. Pivot X, Manikhas A, Zurawski B, et al. CEREBEL (EGF111438): a phase III, randomized, open-label study of lapatinib plus capecitabine versus trastuzumab plus capecitabine in patients with human epidermal growth factor receptor 2-positive metastatic breast cancer. J Clin Oncol. 2015;33(14):1564-1573. doi:10.1200/JCO.2014.57.1794

17. von Minckwitz G, du Bois A, Schmidt M, et al. Trastuzumab beyond progression in human epidermal growth factor receptor 2-positive advanced breast cancer: a german breast group 26/breast international group 03-05 study. J Clin Oncol. 2009;27(12):1999-2006. doi:10.1200/ JCO.2008.19.6618

18. Cancello G, Montagna E, D‘Agostino D, et al. Continuing trastuzumab beyond disease progression: outcomes analysis in patients with metastatic breast cancer. Breast Cancer Res. 2008;10(4):R60. doi:10.1186/bcr2119

19. Extra JM, Antoine EC, Vincent-Salomon A, et al. Efficacy of trastuzumab in routine clinical practice and after progression for metastatic breast cancer patients: the observational Hermine study. Oncologist. 2010;15(8):799-809. doi:10.1634/theoncologist.2009-0029

20. Hammerman A, Greenberg-Dotan S, Feldhamer I, Bitterman H, Yerushalmi R. Second-line treatment of Her2-positive metastatic breast cancer: trastuzumab beyond progression or lapatinib? A population based cohort study. PLoS One. 2015;10(9):e0138229. doi:10.1371/journal.pone.0138229
21. Jackisch C, Welslau M, Schoenegg W, et al. Impact of trastuzumab treatment beyond disease progression for advanced/metastatic breast cancer on survival - results from a prospective, observational study in Germany. Breast. 2014;23(5):603-608. doi:10.1016/j. breast.2014.06.003

22. Montemurro F, Redana S, Viale G, et al. Retrospective evaluation of clinical outcomes in patients with HER2-positive advanced breast cancer progressing on trastuzumab-based therapy in the pre-lapatinib era. Clin Breast Cancer. 2008;8(5):436-442. doi:10.3816/CBC.2008.n.053

23. Negri E, Zambelli A, Franchi M, et al. Effectiveness of trastuzumab in first-line HER2+ metastatic breast cancer after failure in adjuvant setting: a controlled cohort study. Oncologist. 2014;19 (12):1209-1215. doi:10.1634/theoncologist.2014-0227

24. Luque-Cabal M, Garcia-Teijido P, Fernandez-Perez Y, SanchezLorenzo L, Palacio-Vazquez I. Mechanisms behind the resistance to trastuzumab in HER2-amplified breast cancer and strategies to overcome it. Clin Med Insights Oncol. 2016;10(Suppl 1):21-30. doi:10.4137/CMO.S34537

25. Petrelli F, Barni S. A pooled analysis of 2618 patients treated with trastuzumab beyond progression for advanced breast cancer. Clin Breast Cancer. 2013;13(2):81-87. doi:10.1016/j.clbc.2012.11.008

26. Mannocci A, De Feo E, de Waure C, et al. Use of trastuzumab in HER2-positive metastatic breast cancer beyond disease progression: a systematic review of published studies. Tumori. 2010;96 (3):385-391.

27. Ma F, Li Q, Chen S, et al. Phase I study and biomarker analysis of pyrotinib, a novel irreversible pan-ErbB receptor tyrosine kinase inhibitor, in patients with human epidermal growth factor receptor 2-positive metastatic breast cancer. J Clin Oncol. 2017;35 (27):3105-3112. doi:10.1200/JCO.2016.69.6179

28. Li X, Yang C, Wan H, et al. Discovery and development of pyrotinib: A novel irreversible EGFR/HER2 dual tyrosine kinase inhibitor with favorable safety profiles for the treatment of breast cancer. Eur J Pharm Sci. 2017;110:51-61. doi:10.1016/j.ejps.2017.01.021

\section{Publish your work in this journal}

Cancer Management and Research is an international, peer-reviewed open access journal focusing on cancer research and the optimal use of preventative and integrated treatment interventions to achieve improved outcomes, enhanced survival and quality of life for the cancer patient.
The manuscript management system is completely online and includes a very quick and fair peer-review system, which is all easy to use. Visit http://www.dovepress.com/testimonials.php to read real quotes from published authors 